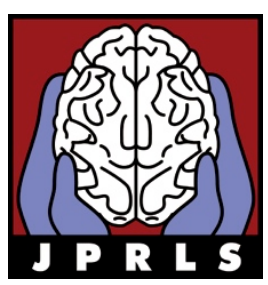

Open Access via www.jprls.org

Journal of Parkinsonism \&

\title{
Report on Positive and Negative Syndrome Scale Workshop
}

\author{
Raza-ur-Rahman* \\ Department of Psychiatry, Dow Medical College, Dow University of Medical Sciences, Karachi, Pakistan
}

It is said, "practice makes perfect". A medic comes well ahead of other professionals who fit this maxim. Owing to the ever changing and ever growing nature of medical sciences, doctors and allied health professionals are in the continuous phase of learning and practicing new techniques and clinical skills.

In recognition of this, the Psychiatry Department of Civil Hospital Karachi (CHK) with the support of International Health Links Funding Scheme (IHLFS) arranged a training workshop for psychiatrists, psychologists, and researchers to refine their skills in administering the very important psychometric tool known as the Positive and Negative Syndrome Scale (PANSS). It is a standardized tool for evaluating positive, negative, and general psychopathology in schizophrenia. This tool requires a structured clinical interview with the patient, during which thirty symptoms are rated by severity. The assessment yields separate scores for the positive syndrome, negative syndrome, composite, and general psychopathology scales. Ratings are assigned on very fine and overlapping findings from the interview. Hence, there are always chances of variation in ratings based on individual raters' understanding of symptomatology. Harmonization and concordance can be achieved through practice and meticulous understanding of fine lines between symptoms.

* Correspondence to: Prof. Raza-ur-Rahman, Head, Department of Psychiatry, Dow Medical College, Dow University of Medical Sciences, Karachi, Pakistan. Email: razaur@yahoo.com.
The objectives of the workshop were to improve understanding of the fine lines of symptomatology that form the basis of ratings, rationalize and harmonize the ratings between raters, improve interviewing skills, build links between institutions, facilitate capacity building of health professionals, and encourage research culture in Pakistan.

Dr. Imran Bashir Chaudhry, consultant psychiatrist and honorary reader at Manchester University, UK facilitated the proceedings. Prof. Raza-ur-Rahman, head of the Psychiatry Department, CHK, chaired the session and Dr. Naeem Siddiqui, consultant psychiatrist at Sindh Institute of Urology and Transplantation (SIUT) graced the event and shared his experiences. The participants were postgraduate trainees, clinical psychologists, and researchers. The workshop was interactive and participants were involved throughout.

A single patient consented for an interview. Two interviewers assessed the patient while participants observed and made their own ratings. After the interview, Dr. Imran Bashir Chaudhry led the discussion and deliberated on each item on the scale. Participants shared their scores and provided justification. Dr. Chaudhry, Prof. Raza-ur-Rehman, and Dr. Siddiqui guided the participants with their experience.

This workshop received encouraging feedback. The live interview and discussion sessions were highly appreciated by the participants. 